others, ${ }^{34}$ emphasise the need for a standardised stimulus and a standardised means of measuring the dermographic response. Only with standardised testing procedures can we estimate with confidence $(a)$ the incidence of dermographism among patients and nonpatients and $(b)$ the relationship between dermographism and other cutaneous responses (for example, chronic urticaria and hyperreactivity to intradermal allergy testing).

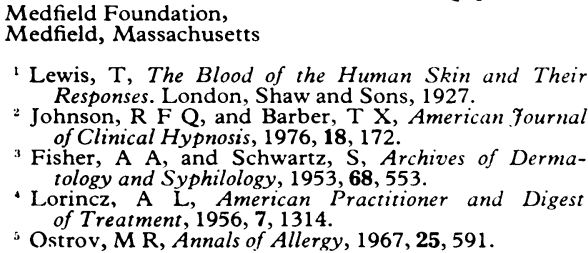
Medfield Foundation,
Medfield, Massachusett

' Lewis, T, The Blood of the Human Skin and Their Responses. London, Shaw and Sons, 1927.

Johnson, R F Q, and Barber, T X, American fournal of Clinical Hypnosis, 1976, 18, 172.

${ }^{3}$ Fisher, A A, and Schwartz, S, Archives of Dermatology and Syphilology, 1953, 68, 553.

Lorincz, A L, American Practitioner and Digest

of Treatment, 1956, 7,

.

RICHARD F Q JOHNSON

\section{Cigarette consumption}

SIR,-Your leading article on chronic bronchitis (29 May, p 1297) states that "in the population as a whole... both men and women continue increasingly to smoke cigarettes." In fact, cigarette consumption has decreased in recent years both by number of cigarettes and weight of tobacco smoked. It is important that this should be widely recognised since it provides encouragement for the many who believe that a gradual reduction in cigarette smoking over a period of 'years is possible.

M DaUBE

London W Executive Director
Action on Smoking and Health

\section{Occult abdominal injury}

SIR,-In your leading article on this subject (22 May, p 1236) the hazards of delayed diagnosis and treatment in blunt abdominal trauma were emphasised, but it was stated that in addition to the use of biochemical and radiological investigations in the diagnosis of patients with symptoms of delayed onset "abdominal paracentesis, especially with peritoneal lavage, may sometimes also help, but the most important factor is repeated clinical examination." It should be said that there must be little or no place for needle paracentesis of the peritoneal cavity in these circumstances because organ penetration may occur and the false-negative diagnostic rate is high. ${ }^{1}$ On the other hand, carefully performed peritoneal lavage is both safe and accurate, a falsepositive rate of $0 \cdot 11^{\circ}$ and a false-negative rate of $0.03^{\circ}$ o being achieved in a large series of cases of blunt abdominal injury. ${ }^{2}$

Early diagnosis of occult abdominal injury before the onset of delayed symptoms is obviously the ideal, and to this end peritoneal lavage should be performed soon after admission to hospital on any patient in whom there exists the possibility of a traumatic intra-abdominal lesion. The presence of blood or bile in the lavage fluid will lead to early laparotomy, whereas a clear return almos certainly excludes significant injury provided the diaphragm is intact. ${ }^{2}$ The lavage cannula (without trocar) is inserted only after careful dissection through the midline infraumbilical abdominal wall to the peritoneum and placement of a purse-string suture, the entry of blood from the wound into the peritoneal cavity thereby being prevented. Even slight blood-staining of the lavage fluid is likely to be significant; it may, for example, result from diffusion into the peritoneal cavity of blood associated with an injured retroperitoneal duodenum or bleeding from the edges of a bowel perforation. The presence of bile may indicate extrahepatic bile duct rupture, a lesion not normally diagnosed until about a week after injury.

It is suggested that greater emphasis should have been placed on the early use of peritoneal lavage in the patient with abdominal trauma, so selecting those who require laparotomy and enabling the occult lesion to be diagnosed and treated within hours rather than days.

Ashford Hospital,

B J PARdy

Fitzgerald, J D, Crawford, E S, and DeBakey, M D, American fournal of Surgery, 1960, 100, 22 Gill, W, et al, British fournal of Surgery, 1975, 62, 121

\section{Myelotoxicity of gold}

SIR,-Dr April G L Kay (22 May, p 1266) recommends frequent blood counts to predict blood dyscrasias. How often is frequent?

Abbots Langley,

Peter Tomson

Herts

**We sent a copy of this letter to $\mathrm{Dr} \mathrm{Kay}$, whose reply is printed below.-ED, $B M \mathcal{F}$.

SIR,--In reply to Dr Tomson the general rule should be that blood is taken each time a patient has an injection of gold and that the subsequent dose should not be given unless the result of the blood count, including platelets, is known to be satisfactory. If there is a downward trend of platelets or granulocytes the count should be repeated, and if the trend persists the next dose of gold should not be given.

Patients who are established on a low dose (for example, sodium aurothiomalate $10 \mathrm{mg}$ a week) may not need such rigorous supervision, but blood counts should be done at least monthly.

APRIL KaY

Arthritis and Rheumatism Council Epidemiology

Research Unit,

Clinical Epidemiology Section,

New Cross Hospital,

London SE14

Status of medical laboratory scientists

SIR,-Mr G P Sutcliffe and his colleagues have pointed out (29 May, p 1339) that the attitude of the Health Departments towards the Institute of Medical Laboratory Sciences' fellowship will inevitably lead both to falling recruitment into the medical laboratory services and to deteriorating standards of patient care. A further consequence of the advice apparently being given to the Departments will be an acceleration of the crumbling demoralisation of Health Service staff.

Apparently not content with having antagonised both junior and consultant medical staff and bringing nurses on to the streets to demonstrate against Health Service policies, the Secretaries of State now appear to be allowing their Departments to antagonise medical laboratory scientists-one of the largest of the professions supplementary to medicine-by the ploy of seeking to determine the fate of a profession without actually consulting it.

This lack of consultation is no new thing Over the past three years this profession has sought to establish a national consultative committee in Scotland under the provisions of the National Health Service (Scotland) Act, 1972. Despite protestations that NHS reorganisation was intended to include increased consultation with staff concerned with patient care-and the general climate of opinion in which, throughout the country, staff participation is now considered a sine qua non for good management-the Scottish Home and Health Department has thought fit to advise the Secretary of State that medical laboratory scientists do not merit the status of a consultative committee. This is despite the recognition, during the same period, of a consultative machinery for another, far smaller, group of medical laboratory workers who can be distinguished only on the grounds of employment under the terms of a different Whitley Council.

Just who advises the Secretaries of State and upon what basis this advice is given is a cause for great concern. Proper consultation could never have led to the present situation in which deterioration of standards of patient care, entry to a profession already undersupplied with qualified senior staff, and the antagonisation of one of the country's largest group of professions supplementary to medicine have all been achieved in one move.

Other Health Service professions should beware. The Health Departments may well have as little regard for their professional standards as their unknown advisers appear to have for medical laboratory scientists.

Aberdeen and NE Scotland Blood

A D FARR

Transfusion Service,

Royal Infirmary,

Aberdeen

\section{Doctors and administration}

SIR,-It has come to our notice that hospital administrators have a higher salary scale than hospital doctors. For example, a district administrator in a large district (the scale is related to number of beds) may earn from $£ 9500$ to $£ 11600$ per annum. This is more than the salary of a full-time consultant, and of course, is very much higher than that of the old group secretary-that is, district administrator-before reorganisation. A similarly unfavourable comparison can be made between the salaries of a sector administrator (hospital secretary) and a registrar. When one considers the training required and the responsibility taken by the doctors in the Health Service this is bound to lead to a sense of injustice and in the long run be bad for the service and patients. It also leads to a comparison with the medical branches of the armed services, where it is of ten necessary to give up clinical medicine to obtain promotion above a certain rank.

It seems probable that Government pay policies will be necessary for the foreseeable future and so it is very unlikely that the relative financial position of consultants who have no private practice can be improved. However, if we are to have such well-paid administrators we should at least reduce their numbers in line with economic realities. It must be clear to 